Meta

Journal des traducteurs

Translators' Journal

\title{
En guise d'introduction... Neurolinguistique, psycholinguistique et traduction
}

\section{Jean-Luc Nespoulous}

Volume 29, numéro 1, mars 1984

Cerveau, langage et traduction

URI : https://id.erudit.org/iderudit/003724ar

DOI : https://doi.org/10.7202/003724ar

Aller au sommaire du numéro

Éditeur(s)

Les Presses de l'Université de Montréal

ISSN

0026-0452 (imprimé)

1492-1421 (numérique)

Découvrir la revue

Citer cet article

Nespoulous, J.-L. (1984). En guise d'introduction... Neurolinguistique, psycholinguistique et traduction. Meta, 29(1), 5-9.

https://doi.org/10.7202/003724ar d'utilisation que vous pouvez consulter en ligne. 


\title{
EN GUISE D'INTRODUCTION... NEUROLINGUISTIQUE, PSYCHOLINGUISTIQUE ET TRADUCTION
}

\author{
JEAN-LUC NESPOULOUS
}

La complexité de la tâche à laquelle fait quotidiennement face un traducteur lorsqu'il tente de restituer en une Langue 2 la substance d'un message initialement formulé dans une Langue 1 n'a plus besoin d'être soulignée. Nombreux sont en effet les auteurs - linguistes ou traducteurs eux-mêmes - qui ont tenté de cerner, aussi rigoureusement que possible, certains des facteurs à l'origine de cette complexité, allant même jusqu'à qualifier parfois de quasiimpossible une telle entreprise (Mounin 1963). Quels que soient les facteurs spécifiques sur lesquels ces auteurs ont plus particulièrement centré leurs investigations et leurs réflexions, il est un thème central, fortement récurrent, nous semble-t-il, dans cette littérature : la difficulté de la traduction trouve une de ses principales sources dans la variation structurale importante qui caractérise les différentes langues du monde.

Notre intention dans le présent volume n'est pas de mettre en doute la véracité des propos tenus par la grande majorité des "théoriciens" de la traduction mais d'essayer de montrer qu'à ces problèmes, déjà amplement documentés, viennent s'en ajouter d'autres, peu étudiés encore, d'ordre psycholinguistique et neurolinguistique, pour employer deux termes qui servent d'appellations génériques à deux disciplines en pleine expansion à l'heure actuelle. En d'autres mots, l'enjeu - fort ambitieux même si notre entreprise demeurera modeste étant donné la relative pénurie de travaux spécifiques en la matière - peut se résumer ainsi : quels sont les processus neuropsycholinguistiques à la base de l'opération de traduction ? Comment le cerveau transforme-t-il en Langue 2 les messages qu'il reçoit - auditivement ou visuellement - en Langue 1 ?

$\tilde{A}$ vrai dire, quiconque s'intéresse aux relations mutuelles existant entre, d'une part, le cerveau et, d'autre part, le langage ou tout autre comportement humain pourrait, sans exagération aucune, prétendre que la fonction essentielle du cerveau réside en permanence dans la "traduction" - ou transformation - d'éléments d'information d'un type A en éléments d'information d'un type B. Dès lors, de la même façon que le cerveau transforme, par exemple, une information visuelle qu'il perçoit en information linguistique (exemple : description d'un paysage), de la même façon qu'il transforme en une chaîne de signes canoniques caractéristiques d'une langue donnée la substance psychique qu'il souhaite transmettre à autrui, il transformerait en une Langue 2 un message initialement formulé dans une Langue 1. Si l'on accepte une telle analogie, le cerveau apparaît alors comme hautement spécialisé dans le " transcodage " d'informations d'un niveau de représentation à un autre (Le Dorze $\&$ Nespoulous dans ce numéro) et la traduction n'est alors envisagée que comme un cas particulier de transcodage dans lequel le point de départ et le point d'arrivée sont tous deux constitués de séquences d'unités linguistiques. 


\section{LA TRADUCTION : INTERROGATIONS ET PERSPECTIVES NEUROLINGUISTIQUES}

Dans nos premières lignes d'introduction - à travers l'utilisation des deux vocables "psycholinguistique " et "neurolinguistique " - nous avons clairement couru le risque que le lecteur considère ces deux domaines d'investigation comme fondamentalement distincts. En fait, il n'en est rien, tout au moins dans notre approche personnelle (et non isolée) du comportement verbal chez l'homme. S'il existe effectivement des psycholinguistes qui s'intéressent aux processus mentaux qui sous-tendent le comportement verbal sans se préoccuper du substrat biologique de la parole et du langage, rares sont les neurolinguistes qui ne s'intéressent qu'aux structures biologiques gouvernant un tel type de comportement sans aborder la nature des processus mis en jeu lors de la production et de la compréhension du langage. En dépit de la complémentarité des deux approches, à laquelle nous sommes personnellement très attachés, nous les dissocierons à des fins strictement didactiques dans ce qui suit.

Du point de vue neurolinguistique, tout d'abord, il convient encore une fois de souligner la pénurie d'études spécifiques ayant trait aux relations mutuelles existant entre le cerveau, d'une part, et l'activité traduisante, d'autre part. Il est toutefois certains travaux, au sein de la neurolinguistique, qui - quoique de façon indirecte - fournissent certaines informations pertinentes sur ce thème. Il s'agit des études visant à rendre compte de l'effet de certaines lésions cérébrales sur le comportement verbal de sujets polyglottes (cf. les articles de Bouton, Lebrun et Paradis dans ce numéro). Si la règle en la matière semble bien résider dans une atteinte équivalente des différentes langues parlées par le patient antérieurement à la survenue du dommage cérébral, un certain nombre d'exceptions ont été rapportées dans la littérature qui font état de l'existence de dissociations notables dans l'utilisation et/ou la récupération des divers systèmes de communication verbale possédés par le patient. Dans ce dernier cas, le neurolinguiste a alors le choix délicat d'interpréter les dissociations mises en évidence sur la base de considérations (1) d'ordre "environnemental " (conditions d'apprentissage, variété de statut des différentes langues ...), ou (2) d'ordre biologique, ce qui le conduit alors à supposer, par exemple, que le cerveau de tel ou tel individu " a décidé " de traiter différemment les langues en présence au point peut-être d'en confier le traitement à des structures cérébrales différentes.

En d'autres termes, selon le type de patients polyglottes auquel on a affaire, on en vient à supposer que le substrat biologique à la base du fonctionnement des différentes langues est identique - d'où la présence de perturbations quantitativement et qualitativement équivalentes dans la manipulation des langues parlées par le patient - ou différent - d'où l'observation, dans certains cas tout au moins, de dissociations importantes dans le comportement verbal des patients selon qu'ils recourent à l'une ou à l'autre des langues qu'ils maîtrisaient parfaitement antérieurement à leur maladie.

Si la plausibilité de l'indépendance neurobiologique des langues est réelle en présence de patients du dernier type évoqué au-dessus, en d'autres termes, si l'on peut concevoir l'existence d'un dispositif biologique (au moins en partie) autonome pour chacune des langues manipulées par un sujet polyglotte qui, en fonction des circonstances, utilise alternativement l'un ou l'autre des systèmes linguistiques qu'il possède à un degré équivalent, une telle thèse ne peut plus être soutenue dès lors que l'on entreprend de "localiser" le substrat biologique d'une activité comme la traduction. L'activité traduisante nécessite l'entrée en interaction des différents systèmes linguistiques requis par la tâche que doit opérer le traducteur. Dès lors, que ces systèmes linguistiques soient ou non logés dans les mêmes zones cérébrales, il demeure clair que le substrat biologique du comportement de traduction requiert l'entrée en jeu de connections nerveuses permettant le va-et-vient indispensable au transcodage en Langue 2 des messages de la Lan- 
gue 1 . On pourrait ainsi prétendre que - chez le bilingue non impliqué dans une tâche de traduction - l'utilisation préférentielle d'une langue dans une situation donnée s'accompagnerait nécessairement de l'inhibition, tant neurophysiologique que neuropsychologique, de l'autre langue connue du patient (une inhibition mal contrôlée débouchant sur les classiques "interférences" bien connues des théoriciens de la traduction). Chez le traducteur, en revanche, une telle inhibition totale serait levée et l'on assisterait à un va-et-vient contrôlé entre les différentes langues impliquées dans le processus de traduction.

\section{LA TRADUCTION : INTERROGATIONS ET PERSPECTIVES PSYCHOLINGUISTIQUES}

D'un point de vue strictement psycholinguistique, à présent, il est au moins deux thèmes d'investigation qui méritent examen pour quiconque s'intéresse à l'activité traduisante.

La première interrogation qui nous paraît présenter un intérêt majeur pour le psycholinguiste a trait au(x) niveau $(x)$ de profondeur au(x)quel(s) le traducteur doit accéder dans la Langue 2 pour restituer convenablement le message qui lui a été adressé en Langue 1. Peut-il, à tout le moins dans certaines circonstances, effectuer le transcodage Langue 1 - Langue 2, sans avoir l'obligation de pénétrer dans les profondeurs des univers de référence constituant la substance du contenu des messages dans les deux langues ? ... ou, au contraire, doit-il toujours s'efforcer d'agir ainsi afin de " produire dans la langue d'arrivée l'équivalent naturel le plus proche du message de la langue de départ " (Nida 1959)?

De plus, et toujours en relation avec les niveaux de profondeur impliqués dans l'activité traduisante, ne peut-on pas envisager l'existence d'une variation importanted'un traducteur à l'autre - dans le recours à tel ou tel niveau ? Si oui, on parviendrait alors à motiver psycholinguistiquement la différence bien connue entre (1) la traduction dite littérale ou mot à mot - asservie à la structure linguistique du message en Langue 1 - et (2) la traduction non littérale qui, de toute évidence, conduit le traducteur à évoluer (A) de la structure de surface en Langue 1 à (B) la substance du contenu de cette structure dans l'univers de référence caractéristique de l'émetteur du message (toujours en Langue 1), puis (C) de l'univers de référence, le plus proche en Langue 2 à (D) l'encodage et la production des structures linguistiques de la Langue 2, structures linguistiques qui se trouvent dès lors énoncées hors de toutes contraintes exercées par les structures linguistiques de la Langue 1 (cf. les articles de Bouton, Paradis, Le Dorze \& Nespoulous dans ce numéro).

La deuxième interrogation fondamentalement psycholinguistique que nous souhaiterions évoquer ici a trait à l'accessibilité des structures linguistiques en Langue 2 lors de l'activité traduisante. Ce thème d'investigation nous paraît particulièrement important dans le contexte de ce type spécifique de traduction qu'est la traduction simultanée. Quelle que soit la complexité intrinsèque - dans la Langue 2 - des structures linguistiques dont le traducteur a besoin pour restituer le message formulé en Langue 1, ce type d'activité traduisante implique la disponibilité immédiate de ces dernières, faute de quoi l'interprète perdrait le fil du discours de l'émetteur initial. Les contraintes temporelles d'une telle activité engendrent vraisemblablement chez ces professionnels des stratégies particulières, passablement différentes - présumons-nous - de celles des traducteurs d'officine, lesquels - comme dans toute tâche de production écrite - s'en trouvent totalement libérés. Notre propos n'est pas d'énoncer ici une liste complète des stratégies auxquelles l'interprète est susceptible de recourir. Nous voudrions simplement expliciter le problème qui nous semble être à l'origine de ces stratégies, quelles qu'elles soient au bout du compte. 
Lors de la traduction du discours en temps réel, l'interprète a la triple contrainte (1) de trouver l'"équivalent le plus naturel "en Langue 2 du message émis en Langue 1 (comme dans toute activité traduisante) et, en plus, (2) d'effectuer cette quête aussi rapidement que possible, tout en (3) prêtant attention au discours subséquent produit par l'émetteur. Lorsque ces trois contraintes - et il y en a certainement d'autres - sont satisfaites, l'interprète effectue son transcodage de façon fluide et harmonieuse. Dans le cas où les deux premières contraintes ne sont pas instantanément satisfaites, l'interprète - qui a compris (on le présume) le sens du message en Langue 1 mais qui ne peut se permettre de poursuivre trop longtemps sa « quête structurale" - se doit (1) de stocker ce fragment d'information en mémoire, (2) de poursuivre son écoute du discours de l'émetteur et (3) de continuer à traduire le discours subséquent en essayant de récupérer le plus rapidement possible l'information laissée en suspens par inaccessibilité structurale temporaire. La production fluide et harmonieuse d'un discours oral est déjà tâche ardue pour le locuteur monolingue. Les nombreuses pauses, hésitations, reprises et phrases avortées qui émaillent tout échantillon de langage conversationnel en sont le témoignage. Que dire alors de la traduction orale en temps réel ? Certainement qu'elle constitue l'une des plus complexes opérations cognitivo-linguistiques qu'il nous soit donné d'observer chez l'être humain, plus complexe, d'une part, du fait des multiples contraintes qui doivent être satisfaites de façon quasi-simultanée et, d'autre part, du fait que les stratégies palliatives "classiques" auxquelles a ordinairement recours un locuteur monolingue (cf. pauses, hésitations ...) ne peuvent pas être utilisées aussi aisément par l'interprète, sous peine - pour ce dernier — de perdre le fil du discours-source. Devant la complexité des processus mis en jeu par une telle activité, on comprend aisément tant la fatigue neuropsychique qui en résulte pour l'interprète que la rareté (!) des études psycholinguistiques sur ce sujet, en dépit du grand intérêt que présente indéniablement un tel thème d'investigation pour les spécialistes de l'étude du comportement verbal humain.

Face à toutes ces interrogations, le présent numéro de Meta s'assigne les deux objectifs suivants : (1) fournir aux spécialistes de la traduction - qu'ils soient théoriciens ou praticiens - certains éléments d'information neurolinguistiques et psycholinguistiques susceptibles de les aider - directement ou indirectement - dans leur réflexion sur les processus et opérations à la base de l'activité traduisante et ce, parallèlement aux éléments d'information fournis par les théoriciens de la traduction évoqués en commençant ; (2) susciter chez les psycholinguistes et les neurolinguistes un engouement de bon aloi pour l'étude de la traduction et des mécanismes qui la sous-tendent.

Dans notre sélection des articles constituant ce numéro, nous avons essayé - autant que faire se pouvait - de garder ces objectifs en point de mire. Ce volume est ainsi organisé en quatre parties :

- Dans une première partie, deux articles présentent la conception - classique (Lecours, Branchereau \& Joanette) et moderne (Joanette \& Lecours) - de la " zone du langage ", en d'autres termes, ce que l'on sait à l'heure actuelle des relations mutuelles du cerveau et du comportement verbal chez l'être humain.

- Dans une deuxième partie, Ch. Bouton, Y. Lebrun et M. Paradis abordent plus spécifiquement la problématique du fonctionnement du cerveau des sujets polyglottes et des traducteurs.

- Dans une troisième partie, plus spécifiquement psycholinguistique, G. Le Dorze et J.-L. Nespoulous présentent différents modèles rendant compte du processus de lexicalisation (ou "mise en mots") chez l'adulte "normal ", l'aphasique et, de façon prospective, chez le traducteur. G. Prideaux et W. Baker, de leur côté, donnent au lec- 
teur un excellent exemple du type de problèmes qu'aborde la psycholinguistique actuelle. Nul doute que la dichotomie fondamentale qui sous-tend le texte de ces auteurs - language-independent psychological processes vs language-specific grammatical rules and structures - est d'une grande pertinence pour quiconque s'intéresse aux divers types de processus à l'oeuvre dans l'activité traduisante, et ce, même si Prideaux et Baker ne se réfêrent pas explicitement à ce type d'activité dans leur exposé.

- Dans une dernière partie, enfin, T. MacNamee et G. Jarema abordent certains aspects et problèmes terminologiques caractéristiques de cette discipline - ancienne quoique récemment baptisée - qu'est la neurolinguistique.

\section{RÉFÉRENCES}

MOUNIN, G. (1963) : les Problèmes théoriques de la traduction, Paris, Gallimard.

NIDA, E.A. (1959): "Principles of Translation Exemplified by Bible Translating", in R.A. Brower (Edit.) On Translation, Cambridge, Mass., Harvard University Press. 